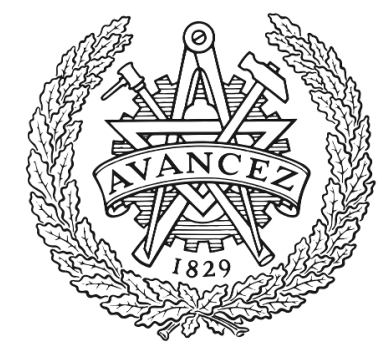

\title{
CHALMERS
}

UNIVERSITY OF TECHNOLOGY

\section{Evaluating IPv6 Connectivity for IEEE 802.15.4 and Bluetooth Low Energy}

Downloaded from: https://research.chalmers.se, 2023-04-26 08:04 UTC

Citation for the original published paper (version of record):

Trelsmo, P., Di Marco, P., Skillermark, P. et al (2017). Evaluating IPv6 Connectivity for IEEE 802.15.4 and Bluetooth Low Energy. 2017 IEEE Wireless Communications and Networking Conference Workshops, WCNCW 2017. http://dx.doi.org/10.1109/WCNCW.2017.7919088

N.B. When citing this work, cite the original published paper. 


\title{
Evaluating IPv6 Connectivity for IEEE 802.15.4 and Bluetooth Low Energy
}

\author{
Patrik Trelsmo*†, Piergiuseppe Di Marco*, Per Skillermark*, Roman Chirikov*, and Johan Östman ${ }^{\dagger}$ \\ * Ericsson Research, Ericsson AB, Stockholm, Sweden. \\ $\dagger$ Department of Signals and Systems, Chalmers University of Technology, Göteborg, Sweden \\ patriktrelsmo@gmail.com, \{piergiuseppe.di.marco; per.skillermark; roman.chirikov\}@ericsson.com, johanos@chalmers.se
}

\begin{abstract}
IPv6 is seen as a key building block for the Internet of Things (IoT). In this work, IEEE 802.15.4 and Bluetooth Low Energy (BLE) protocols are evaluated with respect to service ratio, delay, and energy efficiency under IPv6 traffic. While the performance is terms of delay is similar between the two technologies, BLE outperforms 802.15.4 in terms of service ratio and energy efficiency. Header compression increases all performance indicators but requires a shared context to be set up and managed. IPv6 multicast is also investigated in combination with data link layer broadcast and sequential unicast. Finally, the impact of IPv6 connection establishment and maintenance is evaluated. This is particularly relevant for BLE devices using privacy features. It is shown that the procedures for registering IPv6 addresses and updating contexts affect the delay significantly, but only at very low traffic intensity.
\end{abstract}

\section{INTRODUCTION}

The Internet of Things (IoT) is one of the most widely anticipated paradigm shifts today. Internet connectivity will be available in everyday items such as thermostats, lamps, household appliances and sensors and allow for a wide range of new business opportunities, more efficient use of existing resources such as automated heating, lighting and more.

During the last years there has been an increasing interest in low power wireless access technologies for IoT. These include IEEE 802.15.4 and Bluetooth Low Energy (BLE) among others. To enable interoperability between different medium access technologies, the Internet Protocol version 6 (IPv6) is used [1]. The large address space of IPv6 supports potentially billions of IoT devices. However, adaptation layers are needed to provide header compression and fragmentation of large IP packets, which is critical for low power applications.

This work focuses on 802.15.4 and BLE. The former is seen as a reference standard for home and industrial automation with many large companies being engaged in the development of standards on top of 802.15.4 [2]. BLE is interesting because of the large number of devices shipped and the activity regarding standardization and development of new applications [3]. Internet connectivity for 802.15.4 is provided with the adaptation layer IPv6 over Low-Power Wireless Personal Area Networks (6LoWPAN), released in 2007 [4]. For BLE, there exists a similar adaptation layer called 6LoBTLE, released in October 2015 [5]. While solutions that enable IPv6 for both BLE and 802.15.4 exist, there is still much standardization work to improve IPv6 connectivity for low power radio technologies.
For BLE and 802.15.4 there are many performance studies in terms of delay [6]-[9] and energy efficiency [7], [9]-[13]. Some works in the literature only consider a single device disturbed with interference [11], while others consider larger networks of devices [6], [12]. There are also studies that only measure the performance of a single device without any interference [7], [10]. In [13], IPv6 over BLE and 802.15.4 is studied, but only for single devices in a controlled environment. To the best of our knowledge, there are no studies where the performance of these radio access technologies is benchmarked in a real use case and where IPv6 traffic is considered.

This paper provides a comprehensive evaluation of BLE against 802.15.4 for a home automation use case. More specifically, the performance of BLE and 802.15.4 is evaluated in the presence of IPv6 connectivity with and without 6LoWPAN (or 6LoBTLE) header compression. Issues, such as how to handle IPv6 multicast destinations and how to establish and maintain IPv6 networks are also addressed.

\section{Short Range Radio Technologies For IoT}

This section introduces radio technologies and protocols for IoT, starting with a comparison between 802.15.4 [14] and BLE [15]. Moreover, a brief introduction to IPv6 [16] and 6LoWPAN [5], [17] is provided.

\section{A. IEEE 802.15.4 vs. BLE}

802.15.4 is one of the earliest standards developed for low power wireless networks, with a wide range of IoT use cases, spanning from industrial and office automation to the connected home. It has been adopted with some modifications also by a number of other protocol stacks, including ZigBee, WirelessHART, and ISA-100 [2]. BLE is an open wireless technology standard for short range communications, designed as a complement to classic Bluetooth. The use case for Bluetooth has for a long time been the wireless connection between a mobile phone and a headset. BLE, which was released in 2010 as part of Bluetooth 4.0, constituted a successful step towards expanding the Bluetooth ecosystem for IoT.

802.15.4 supports star and peer-to-peer topologies that can be extended as generic mesh whose nodes are clustered with a local coordinator and connected via multi-hop to a global coordinator. BLE is limited to star and single-hop peer-to-peer 
networks. However, mesh networking functionality is expected to be standardized in future Bluetooth specifications.

802.15.4 operates in the $2.4 \mathrm{GHz}$ industrial, scientific and medical (ISM) frequency band as well as sub-GHz ISM bands. Only the $2.4 \mathrm{GHz}$ operation is mandatory and the default mode uses 16 channels, each with $2 \mathrm{MHz}$ bandwidth, Direct Sequence Spread Spectrum and O-QPSK modulation with $250 \mathrm{kbps}$ bit rate. BLE uses only the $2.4 \mathrm{GHz}$ band and the available spectrum is divided into 40 channels of $1 \mathrm{MHz}$ bandwidth; three advertising channels and 37 data channels. Furthermore, BLE employs Gaussian Frequency Shift Keying and the over the bit rate is $1 \mathrm{Mbps}$. Additional rates will likely be supported by future releases of the standard.

The medium access control (MAC) mechanism presents fundamental differences between the two technologies. 802.15.4 has two modalities: a slotted one based on a superframe structure with Carrier Sensing Multiple Access with Collision Avoidance (CSMA/CA) and time division multiple access; an unslotted modality with only CSMA/CA. We focus on the second option in this study since it guarantees scalability for large networks. BLE works with both connectionless and connection-oriented data transfer modes. In the former mode, BLE uses a basic ALOHA scheme over three advertising channels. However, only small payloads (31 bytes) are supported according to the current specifications. The latter uses channel hopping data transmissions. On top, the logical link control and adaptation protocol (L2CAP) performs segmentation and reassembly of large packets in BLE. We focus on the connection-oriented option since it enables IPv6 packet transactions.

\section{B. IPv6 networking}

IPv6 is a network layer defined originally by the Internet Engineering Task Force to extend the functionalities of the Internet Protocol. Here we focus on header, auto-configuration of addresses and neighbor discovery. No routing protocols are studied and security is provided by higher layers.

The IPv6 address consists of 128 bits and can be classified into three groups; unicast, multicast and anycast. Stateless address auto-configuration is performed by using a link-local prefix and appending a unique EUI-64 identifier. For a global unicast address, a device must first obtain the network prefix through Neighbor Discovery, then insert the EUI-64 to create the address [16].

The procedure for connecting a device to an IPv6 network comprises of the following steps: the device first transmits a router solicitation, containing the link layer address of the originating device. Upon reception, an IPv6 router will respond with a router advertisement that contains parameters for the IPv6 network such as current hop limit, prefix information, context information and more.

The registration of generated IPv6 addresses is done by the device sending a neighbor solicitation message per address. The router maintains a mapping from link layer address to IPv6 address for all devices in the network in order to perform duplicate address detection without querying other devices.
Neighbor advertisement messages are returned with the result of the duplicate address detection.

During this process, timers are being set in many places. For instance, when registering an IPv6 address, a lifetime for the address is chosen. When this lifetime expires, the address is no longer valid and it must be re-registered before it can be used again. The maximum registration lifetime is approximately 45 days, so this is a rare event. But, if for some reason, the maximum registration lifetime cannot be used, re-registration might happen more frequently.

\section{Adaptation layers}

The goal of the adaptation layers 6LoWPAN and 6LoBTLE is to enable IPv6 packets to be transmitted over low power, lossy networks with devices that are sleeping (i.e. transceiver turned off) most of the time.

To achieve this, the IPv6 header is shortened by not transmitting all fields and by making use of how IPv6 addresses are created to perform stateless or context-based compression of IPv6 addresses. Each device maintains a data structure for context information used for compression of IPv6 addresses. The structure is called the context table. Each context entry in the table is associated to a context ID, the prefix/partial address, a compression bit and the valid lifetime [18]. Fragmentation and reassembly functionality is only provided in 6LoWPAN for 802.15.4 networks. In 6LoBTLE no fragmentation is required since it is performed by the L2CAP layer.

\section{SCENARIO AND ASSUMPTIONS}

The performance assessment is based on a common deployment, traffic model implementation, and higher layer protocol assumptions.

The scenario consists of a $12 \mathrm{~m}$ x $10 \mathrm{~m}$ single-family home, as depicted in Fig. 1. Sensor and actuator devices are deployed within the building, consisting of window and temperature sensors, asset tags, as well as light switches and light bulbs. In total, 78 devices are deployed, including the central gateway that acts as an 802.15.4 coordinator or a BLE central device. Devices are described with packet size and mean packet arrival interval in Tab. I. All devices are assumed to be stationary.

The central gateway is mains-powered having its radio receiver always active. It implements the application logic, for example a local cloud, and it has Internet connectivity for messages directed to an external cloud. The sensor devices are battery powered and are typically in sleep mode with their transceiver turned off. When a sensor report is generated, the radio components are activated and the communication process starts. To keep energy consumption down, sensor devices are not reachable by the central gateway except when new sensor report triggers the devices to become active. Actuator devices (lamps) are waiting for application layer actuation commands to act upon, meaning that they must be reachable by the central gateway.

Application layer messages are randomly generated with exponentially distributed inter-arrival time. A command from a light switch is first transmitted to the central gateway, in which 


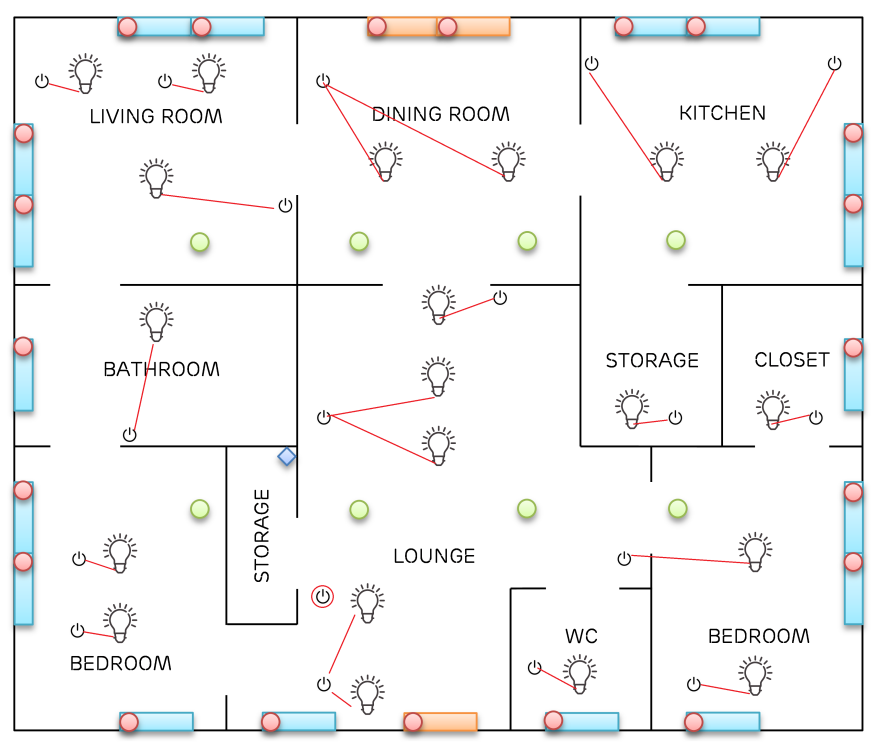

Fig. 1. Deployment of devices for the simulation scenario used. Red lines represent logical connections between lamps and lamp switches. All communications go through the central device, meaning that a command from a lamp switch is sent to the gateway where it is forwarded to the corresponding lamp(s) according to the logical connections.

it is processed on application layer resulting in a command from the central device to one or several associated light bulbs. Accordingly, in this setup all data transfer takes place via the central gateway and there is no direct communication between sensors and actuators. Because of the limited number of simultaneous connections currently supported for BLE, it is assumed that devices disconnect when neither side has more data to transmit.

In the default scenario, the traffic load is approximately $120 \mathrm{bps}$ at the application layer. A scenario with low load (12 bps) and three scenarios with increasing load (240, $480 \& 960$ bps) are considered to measure the sensitivity to varying traffic load.

Radio propagation is modeled using the indoor propagation model from [19], which assumes $6 \mathrm{~dB}$ internal wall loss and a distance dependent propagation loss of $0.5 \mathrm{~dB}$ per meter. Shadow fading is assumed to be uncorrelated across links and is modeled as a log-normal, distributed random variable with zero mean and a standard deviation of $6 \mathrm{~dB}$. The central gateway antenna gain is $0 \mathrm{~dB}$ while the sensor and actuator antenna gains are $-4 \mathrm{~dB}$. For a receiver that is turned on and tuned to the correct channel, the packet reception probability is determined by the signal-to-noise-and-interference power ratio (SINR) at the receiver, as well as the physical layer performance of the modulation and coding scheme used.

\section{Performance Evaluation}

\section{A. Performance indicators}

The performance of the two technologies was compared by means of extensive system level simulations in terms of traffic

\footnotetext{
${ }^{1}$ Not included in figure - uniformly distributed across the simulated area.
}

TABLE I

DESCRIPTION OF THE SIMULATED DEVICES.

\begin{tabular}{ccccc} 
Icon & $\begin{array}{c}\text { Device } \\
\text { Type }\end{array}$ & $\begin{array}{c}\text { \# of } \\
\text { Devices }\end{array}$ & $\begin{array}{c}\text { Payload } \\
\text { Size }\end{array}$ & $\begin{array}{c}\text { Packet } \\
\text { Interval }\end{array}$ \\
\hline 垱 & Lamp & 20 & $1 \mathrm{~B}$ & $5 \mathrm{~min}$ \\
$\sqcup$ & Switch & 18 & $1 \mathrm{~B}$ & $5 \mathrm{~min}$ \\
0 & Window Sensor & 21 & $1 \mathrm{~B}$ & $5 \mathrm{~s}$ \\
& Temp. Sensor & 8 & $4 \mathrm{~B}$ & $1 \mathrm{~min}$ \\
& Asset Tag ${ }^{1}$ & 10 & $1 \mathrm{~B}$ & $1 \mathrm{~s}$ \\
& Gateway & 1 & - & -
\end{tabular}

service ratio, delay, and energy consumption. The service ratio was measured on the application layer by comparing the amount of generated and served traffic in terms of bits. The delay was also measured on the application layer, by associating each transmitted packet with the time of generation and comparing it to the time of reception. Single hop delay was measured for all transmissions in the network, while end-toend delay was only measured for traffic from light switches to the corresponding lamps, via the central gateway. Finally, the energy efficiency, or battery life expectancy, was considered. The energy consumption was based on measurements from the physical layer in the simulator. Values are taken from the data sheets for Texas Instruments CC2630 and CC2640 micro-controllers, assuming 3V CR2032 battery with $200 \mathrm{mAh}$ capacity [20], [21].

\section{B. Results for IPv6 over 802.15.4 and BLE}

A first set of simulations was run with IPv6 in steady state operation. In Figure 2, the service ratio of 802.15.4 and BLE is reported as a function of the traffic load with and without 6LoWPAN. In both cases, BLE serves a higher load with more reliability than 802.15.4. This is mainly due to the higher overthe-air rate of BLE and the fact that data transmissions take place on dedicated channels that do not interfere with advertisements while in 802.15.4 all transmissions take place in the same frequency band leading to collisions and congestion.

The main reason for packet loss in 802.15.4 is because of the retry limit, not the channel access failure. This can be explained with the hidden terminal issue. If there is a collision and retransmission is performed, the CSMA parameters are reset, which limits the maximum backoff to $2.24 \mathrm{~ms}$ (140 symbols). The transmission of the packets in the simulation (119 bytes) takes approximately $3.8 \mathrm{~ms}$, which means that the probability of colliding on the next transmission again is high since both devices will attempt transmitting again within 2.24 ms of the ACK wait duration.

The reason for losses in BLE is because of congestion on the advertising channels. When advertising, a supervision timer is set to $200 \mathrm{~ms}$ in the simulations. If no connection is established within that time, the receiving device is assumed to be (temporarily) unavailable and all packets destined for that device are discarded. For the default scenario, the random delay introduced between advertising events (uniformly distributed between 0 and $10 \mathrm{~ms}$ ) manages to solve collisions. 
If a connection is established, the transfer of data takes place on a dedicated channel without interference.

Looking at the traffic service ratio in Fig. 2 it is clear that introducing header compression increases the service ratio. Especially for 802.15.4, the service ratio is increased substantially for higher traffic loads. Because all transmissions take place on the same channel, reducing the size of packets leads to less congestion, thus increasing the service ratio. For BLE, the size of the advertisements does not change, neither does the frequency of transmissions. Therefore the congestion on the advertising channels is only affected by the reduced duration of data transmissions that gives less queued transmissions.

The end-to-end delay between a light switch and the corresponding light(s) is reported in Fig. 3. The values are very similar for the two technologies. The delay in each hop for BLE contains establishing a new connection by advertising, receiving a connection request and then transmitting the data in the first connection event. For most of the transmissions this takes approximately $4 \mathrm{~ms}$, while some transmissions take an additional 20-30 ms because of a lost advertisement or connection request. For 802.15.4, the CDF shows signs of more delay jitter, since the randomness of the backoff period in the CSMA/CA spreads the delay. While 802.15.4 does not require connections to be established, the actual bit rate is lower and the delay becomes higher. This can be connected to the large packet sizes, noticing that the relative overhead of establishing a connection in BLE is reduced as the amount of data increases. The end-to-end delay is slightly higher than twice the single-hop delay. Since IPv6 packets are not broadcast on the MAC layer in this scenario, the central device must establish a connection to each lamp in succession, as follows; the central device receives a command from a switch and shall relay this to the two registered lamps. It first connects to one of the lamps and transmits the command. When acknowledged (or failed) it terminates the connection with the first lamp and connects to the second. Therefore the second lamp also experiences the delay of the first.

As can be seen in Fig. 3, header compression only reduces the delay marginally. Even if the number of packets transmitted over the air in BLE is reduced after introducing header compression, the overhead of setting up a connection is not reduced. This results in the minor reduction of 2 to 3 $\mathrm{ms}$ in the end-to-end delay. For 802.15.4, the largest delay is introduced by the overhead and transmitting bits over the air, since the packet size is large enough to carry the generated IPv6 packets without fragmentation and there is no need to set up connections. The reduction of 2 to $4 \mathrm{~ms}$ is explained partly because of reduced contention and thereby backoff, but mainly by the lower number of bits being transmitted over the air.

The expected battery lifetime for sensors is reported in Fig 4. BLE devices consume less energy than 802.15.4 devices. This is explained by the same reasoning as for the delay, since BLE devices transmit with four times the bit rate of 802.15.4 and the packets are large (low overhead of establish-

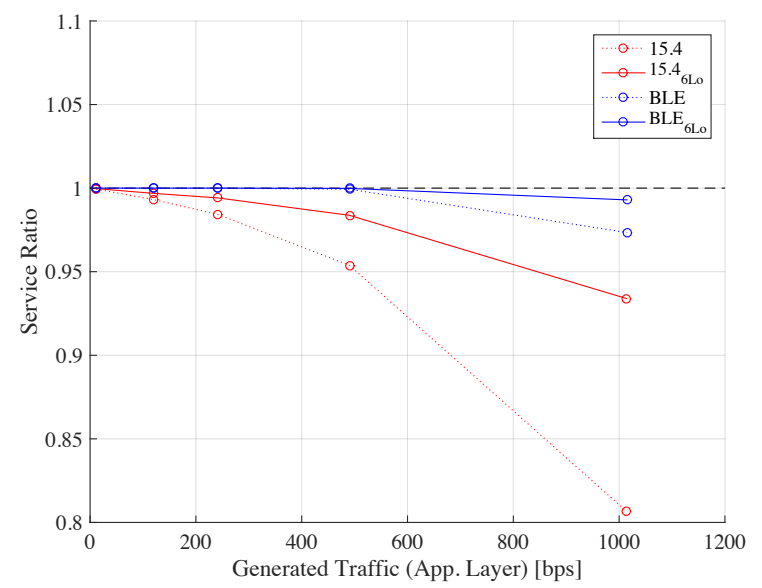

Fig. 2. Traffic Service Ratio as a function of traffic load. The default scenario corresponds to the second data points (120 bps).

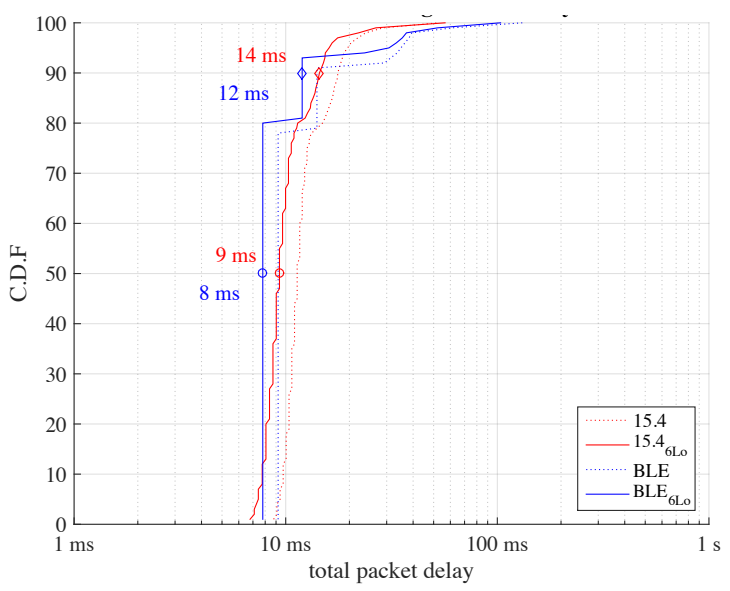

Fig. 3. End-to-end delay under default traffic load. Calculated on application layer with and without IPv6 header compression.

ing connections), BLE devices spend less time in active states. The introduction of header compression reduces the amount of traffic on the link layer because of the reduced IPv6 overhead. The same 6LoWPAN settings were used for both BLE and 802.15.4 which is reflected in the similar increase of energy efficiency, as can be seen in Fig. 4. The median lifetime is increased by $23.7 \%$ for BLE and by $33.5 \%$ for 802.15 .4 . Again the difference in the performance improvement relates to bit rate and packet size. The reduction of the active time when reducing IPv6 overhead is more visible in 802.15.4 due to the lower transmission rate.

\section{Results with IPv6 multicast}

IPv6 Multicast addresses are used for transmitting one packet to several destinations without replicating the transmission on the IP layer for the individual destinations. This type of address can be used to reduce transmissions for applications such as lighting, where one switch controls multiple lamps. At the MAC layer, transmission of multicast packets can be done by either sending a single broadcast transmission or by 


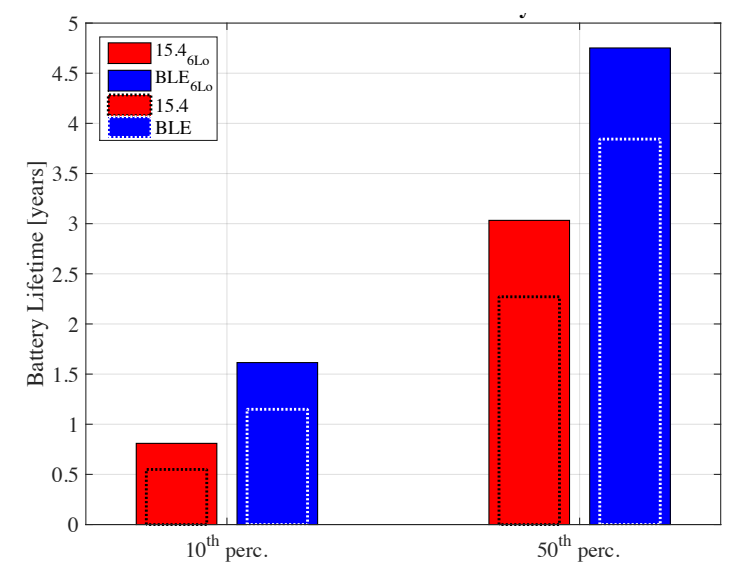

Fig. 4. Expected battery lifetime for sensors under default IPv6 traffic load, with and without header compression.

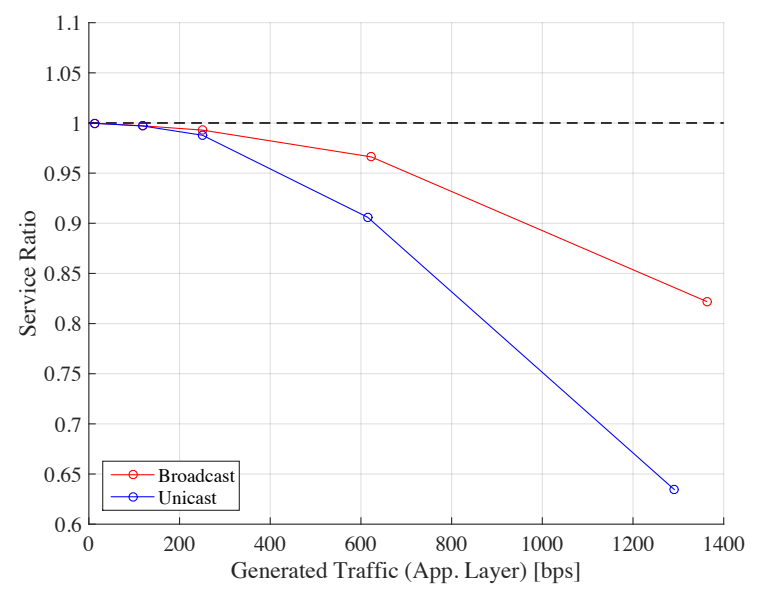

Fig. 5. Traffic Service Ratio as a function of traffic load. Note that this is for 802.15 .4 devices only.

sequentially transmitting copies of the original packet to one receiver at a time.

In the following, we show results for the two methods for delivering IPv6 multicast traffic on top of 802.15.4. For the considered version of BLE [15], the only way to transmit IPv6 packets is by setting up a connection to another device and unicast to each destination. In fact, broadcast advertising packets in BLE have maximum payload size of 31 bytes and no fragmentation is provided.

The simulated use case is the home automation scenario presented in Sec. III, but an additional light switch that controls all lights is included. The lights are assumed to be registered to multicast IPv6 addresses, even if there is only one light in the multicast group. Then the scenario is simulated when the multicast transmissions are broadcast on the MAC layer and when they are transmitted to each of the registered listeners one at a time, i.e. unicast.

The service ratio is reported in Fig. 5. Although broadcast transmissions cannot be acknowledged, the service ratio is higher when the multicast transmissions are broadcast on the

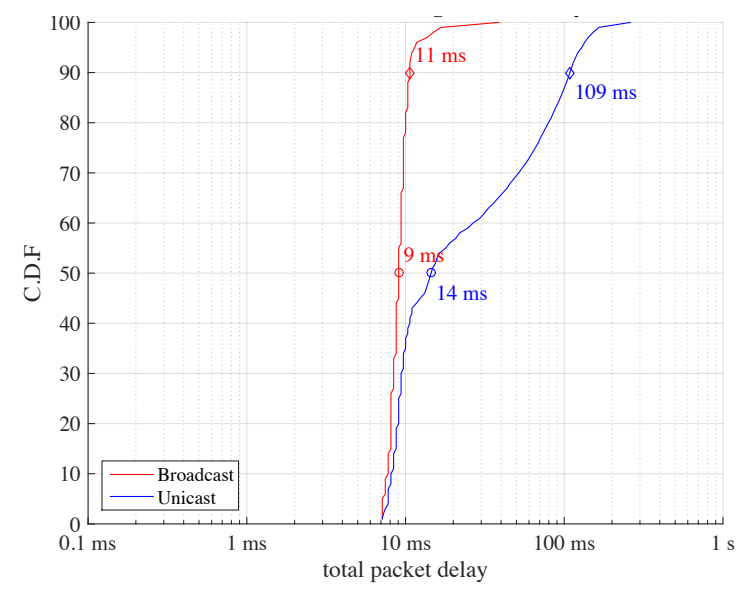

Fig. 6. End-to-end delay under default traffic load for 802.15.4 when either broadcasting or unicasting on the MAC layer.

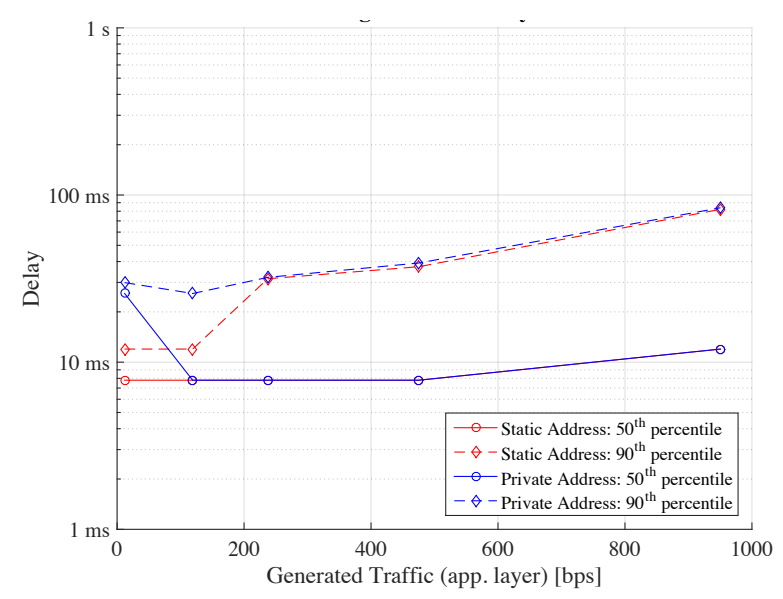

Fig. 7. End-to-end delay as a function of traffic load for BLE using static or resolvable private link layer addresses.

MAC layer than when they are unicast. One explanation for this is that the channel quality is high and that retransmissions due to bad channel conditions are rare. Transmitting fewer packets then reduces the risk of collisions. Reducing the traffic load also reduces collisions due to hidden terminals.

The end-to-end delay for lamps is shown in Fig. 6. As expected, broadcasting results in a lower delay for successful transmissions than unicasting. This is explained simply by noting that in broadcasting mode, only one transmission is sent over the air, regardless of the number of destinations.

In previous works, such as [22], it has been shown that broadcasting is not useful in a generic mesh scenario. When considering routing and a mesh topology, the overhead of having many devices receiving the same data outweighs the benefits from only having to transmit each packet once. However, in the scenario considered for this study, with star topology and good coverage, it is instead shown than there are benefits of using link layer broadcasting for IPv6 multicast packets, since it can reduce delay and increase service ratio. 


\section{Results with IPv6 connection maintenance}

IPv6 connection establishment and maintenance procedures are performed when a node joins the network and whenever an IPv6 address, or context information, need to be registered or updated. For the privacy of BLE devices, link layer addresses may be changed every 15 minutes [15]. When changing a link layer address, RFC 7668 requires new non link-local IPv6 addresses to be registered [5].

In order to study the effects of this, simulations of two cases were considered. One where the devices use static link layer addresses, and one where private link layer addresses are used and neighbor discovery has to be performed every time a device wakes up after 15 minutes from the previous transmission, in order to register the new IPv6 address.

There was no significant difference found between the two scenarios in terms of traffic ratio. For low traffic load, the network congestion is low and the additional traffic due to the IPv6 connection maintenance is served without affecting the service ratio noticeably. For high traffic load, the overhead from IPv6 connection maintenance is low compared to the total traffic and it does not affect the resulting service ratio. Figure 7 shows the end-to-end delay for traffic from lamp switches for various traffic load values. For low intensity, the overhead in the private address case for registering an IPv6 address and refreshing context entries $(\approx 18 \mathrm{~ms})$ degrades the performance significantly. In the first traffic setting, a new address is registered at every packet transmission in average. The delay converges as the traffic load increases. When the load increases, the fraction of transmissions that are queued behind the process of establishing an IP connection decreases.

\section{CONCLUSIONS}

The performance of IPv6 over 802.15.4 and BLE was evaluated for a realistic home automation use case. It was found that BLE outperforms 802.15.4 in terms of service ratio because of the higher bit rate and dedicated data channels. In terms of delay, the performance is similar in the default scenario, but the delay for BLE is more sensitive to the traffic load than 802.15.4. It was finally shown that BLE devices use less energy to run the same network setup and that as a result the BLE devices have a longer expected lifetime. This is directly related to the high bit rate which means that BLE devices spend less time in active states than 802.15.4 devices.

When 6LoWPAN header compression was implemented, the service ratio was improved mainly for 802.15.4. BLE also benefited from reduced overhead, but since the bit rate is lower for 802.15.4, each elided bit improved the energy efficiency for 802.15.4 more than for BLE. For 802.15.4, in contrast to previous works, it was found that the service ratio may improve by broadcasting messages with IPv6 multicast destinations, despite the inability to acknowledge broadcasts.

Finally, the impact of IPv6 connection maintenance was studied. In the default scenario, approximately $15-20 \%$ of transmissions from light switches were affected by the extra messages exchanged when private BLE addresses are used. For low traffic conditions, the cost of enabling 6LoBTLE appears to be high compared to the benefits in terms of header compression. For high traffic conditions, the benefits of 6LoBTLE are significant.

\section{REFERENCES}

[1] A. Williams, "IoT gets smart with Bluetooth." Electronics Weekly, no. 2642, p. 12, 2015.

[2] A. Willig, "Recent and emerging topics in wireless industrial communications: A selection," IEEE Transactions on Industrial Informatics, vol. 4, no. 2, pp. 102-124, May 2008.

[3] "ABI Research Predicts Wireless Connectivity Gap to Widen as Bluetooth Enabled Device Shipments Reach 19 Billion over the Next Five Years," Telecommunications Weekly, p. 45, December 2015.

[4] G. Montenegro, N. Kushalnagar, J. Hui, and D. Culler, "Transmission of IPv6 packets over IEEE 802.15.4 networks," Internet Requests for Comments, RFC Editor, RFC 4944, September 2007.

[5] J. Nieminen, T. Savolainen, M. Isomaki, B. Patil, Z. Shelby, and C. Gomez, "IPv6 over BLUETOOTH(R) Low Energy," Internet Requests for Comments, RFC Editor, RFC 7668, October 2015.

[6] P. D. Marco, C. Fischione, F. Santucci, and K. H. Johansson, "Modeling IEEE 802.15.4 networks over fading channels," IEEE Transactions on Wireless Communications, vol. 13, no. 10, pp. 5366-5381, Oct 2014.

[7] K. Mikhaylov, N. Plevritakis, and J. Tervonen, "Performance analysis and comparison of Bluetooth Low Energy with IEEE 802.15.4 and SimpliciTI," Journal of Sensor and Actuator Networks, vol. 2, no. 3, pp. 589-613, 2013.

[8] M. Petrova, J. Riihijarvi, P. Mahonen, and S. Labella, "Performance study of IEEE 802.15.4 using measurements and simulations," in IEEE Wireless Communications and Networking Conference (WCNC), April 2006, pp. 487-492.

[9] H. Wang, M. Xi, J. Liu, and C. Chen, "Transmitting IPv6 packets over Bluetooth Low Energy based on BlueZ," in 15th International Conference on Advanced Communication Technology (ICACT), Jan 2013, pp. 72-77.

[10] J. Nieminen, C. Gomez, M. Isomaki, T. Savolainen, B. Patil, Z. Shelby, M. Xi, and J. Oller, "Networking solutions for connecting Bluetooth Low Energy enabled machines to the Internet of Things," IEEE Network, pp. 83-90, 112014.

[11] E. Casilari, J. M. Cano-Garca, and G. Campos-Garrido, "Modeling of current consumption in 802.15.4/ZigBee sensor motes," Sensors, vol. 10, no. 6 , pp. 5443-5468, 2010.

[12] W. Du, D. Navarro, and F. Mieyeville, "Performance evaluation of IEEE 802.15.4 sensor networks in industrial applications," International Journal of Communication Systems, vol. 28, no. 10, pp. 1657-1674, 2015.

[13] M. Siekkinen, M. Hiienkari, J. Nurminen, and J. Nieminen, "How low energy is Bluetooth Low Energy? Comparative measurements with ZigBee/802.15.4," in IEEE Wireless Communications and Networking Conference Workshops (WCNCW). IEEE, April 2012, pp. 232-237.

[14] IEEE Standard for Local and metropolitan area networks-Part 15.4: Low-Rate Wireless Personal Area Networks (LR-WPANs), IEEE 802.15.4-2011, 2011, [Online]. Available: https://standards.ieee.org/ about/get/802/802.15.html.

[15] Bluetooth Core Specification 4.1, Bluetooth SIG, 2014, [Online]. Available: https://www.bluetooth.com/specifications/adopted-specifications.

[16] R. Graziani, IPv6 Fundamentals: A Straightforward Approach to Understanding IPv6. Cisco Press, 2013.

[17] J. Hui and P. Thubert, "Compression format for IPv6 datagrams over IEEE 802.15.4-based networks," Internet Requests for Comments, RFC Editor, RFC 6282, September 2011.

[18] Z. Shelby, S. Chakrabarti, E. Nordmark, and C. Bormann, "Neighbor Discovery optimization for IPv6 over Low-power Wireless Personal Area Networks (6LoWPANs)," Internet Requests for Comments, RFC Editor, RFC 6775, November 2012.

[19] C. Tornevik, J. E. Berg, F. Lotse, and M. Madfors, "Propagation models, cell planning and channel allocation for indoor applications of cellular systems," in IEEE Vehicular Technology Conference, 1993, pp. 867-870.

[20] CC2630 SimpleLink 6LoWPAN, ZigBee Wireless MCU, Texas Instruments, 2015, [Online.] Available: http://www.ti.com/product/CC2630.

[21] CC2640 SimpleLink Bluetooth Smart Wireless MCU, Texas Instruments, 2015, [Online.] Available: http://www.ti.com/product/CC2640.

[22] X. Wang, "Multicast for 6LoWPAN wireless sensor networks," IEEE Sensors Journal, vol. 15, no. 5, pp. 3076-3083, 2015. 